\title{
Comparative genomics reveals multiple pathways to mutualism for tick-borne pathogens
}

\author{
Svetlana Lockwood ${ }^{1}$, Kelly A. Brayton ${ }^{2,3}$ and Shira L. Broschat ${ }^{1,2,3 *}$
}

\begin{abstract}
Background: Multiple important human and livestock pathogens employ ticks as their primary host vectors. It is not currently known whether this means of infecting a host arose once or many times during evolution.

Results: In order to address this question, we conducted a comparative genomics analysis on a set of bacterial pathogens from seven genera - Borrelia, Rickettsia, Anaplasma, Ehrlichia, Francisella, Coxiella, and Bartonella, including species from three different host vectors - ticks, lice, and fleas. The final set of 102 genomes used in the study encoded a total of 120,046 protein sequences. We found that no genes or metabolic pathways were present in all tick-borne bacteria. However, we found some genes and pathways were present in subsets of tick-transmitted organisms while absent from bacteria transmitted by lice or fleas.
\end{abstract}

Conclusion: Our analysis suggests that the ability of pathogens to be transmitted by ticks arose multiple times over the course of evolution. To our knowledge, this is the most comprehensive study of tick transmissibility to date.

Keywords: Tick-borne pathogens, Phylogenomic network, Comparative genomics

\section{Background}

Based on World Health Organization estimates, in recent years the confounding factors of climate change and human demographics have had a significant impact on the increase in vector-borne diseases [1]. A number of severe diseases are caused by different vector-borne bacteria. According to the Centers for Disease Control and Prevention, Borrelia burgdorferi, a member of the order Spirochaetes, infects 300,000 people with Lyme disease in the United States annually [2]. Bites by ticks carrying the highly infectious bacterium Francisella tularensis result in the potentially fatal disease tularemia [3, 4], and more than 20 types of fever and typhus worldwide are attributed to tick-borne members of the spotted fever group of Rickettsia [5].

*Correspondence: shira@eecs.wsu.edu

1 School of Electrical Engineering and Computer Science, Washington State University, P.O. Box 642752, 99164-2752 Pullman, USA

2 Department of Veterinary Microbiology and Pathology, Washington State University, P.O. Box 647040, 99164-7040 Pullman, USA

Full list of author information is available at the end of the article
Vector-borne pathogens exhibit remarkable evolutionary adaptations, which allow them to exploit an array of vector hosts even within one genus. Borrelia hermsii, the agent of tick-borne relapsing fever, uses as its vector soft-bodied ticks of the genus Ornithodoros [6] while Borrelia burgdorferi, the causative agent of Lyme disease, is transmitted by the hard-bodied tick Ixodes scapularis [7]. Interestingly, these host ticks are widely divergent, separated by millions of years of evolution [8]. Fleas are well-established vectors for Bartonella henselae [9-12] while Bartonella quintana utilizes lice [13]. Even more remarkably, members of the genus Rickettsia use a range of different vectors - fleas ( $R$. typhi, R. felis), lice ( $R$. prowazekii), mites ( $R$. akari), and ticks (R. parkeri, $R$. japonica, $R$. helvetica, and others).

Among pathogen vectors, ticks are of particular concern as they play a prominent role in transmission of pathogens to humans and livestock. In the US, for example, incidents of Lyme disease almost tripled in the past two decades resulting in $\$ 0.7-\$ 1.3$ billion in direct medical costs [14-17]. In Tanzania, economic losses resulting not 
only from direct production losses, but also from treatment and control costs associated with tick-borne diseases (TBDs) alone, are estimated to be 364 million USD annually [18]. Given the significant impact of TBDs both on human health and on economic activities, it is important to understand the biological mechanisms of pathogen transmissibility. However, bio-molecular determinants of tick-borne transmission are still largely unknown.

An intriguing question is whether the ability for a bacterial pathogen to be tick transmitted evolved one or more times. We hypothesized that there may exist a common molecular mechanism among all tick-associated bacterial pathogens. Identification of such a mechanism across different species would suggest a path for the development of transmission blocking strategies. We test our hypothesis by undertaking a comparative genomics approach to analyze bacteria in the genera Borrelia, Rickettsia, Anaplasma, Ehrlichia, Francisella, Coxiella, and Bartonella, conducting the most comprehensive study examining tick transmissibility to-date.

\section{Methods}

To explore the bio-molecular evidence of tick transmissibility we collected data for all vector-borne bacteria for which full genome sequences are available and a vector is known. These included organisms from seven genera Borrelia, Bartonella, Rickettsia, Anaplasma, Ehrlichia, Coxiella, and Francisella (Table 1). From these microorganisms a set was selected for the most prevalent arthropod vectors - ticks, fleas, and lice. Genomes for $R$. akari, Orienta tsutsugamushi, and Neorickettsia sennetsu were excluded from the analysis due to the lack of sufficient representation of their particular vectors - chiggers, mites, and trematodes, respectively. We did not include species for which vectors were either unconfirmed or unknown. In addition, a few Rickettsia genomes and one Borrelia genome were excluded because annotation for them does not exist in the KEGG organism

Table 1 Numbers of genomes examined and their vector association

\begin{tabular}{lllll}
\hline Genus & \multicolumn{3}{c}{ Vectors } & Number of genomes \\
\cline { 2 - 4 } & Tick & Louse & Flea & \\
\hline Anaplasma & 6 & 0 & 0 & $\mathbf{6}$ \\
Bartonella & 0 & 2 & 4 & $\mathbf{6}$ \\
Borrelia & 17 & 1 & 0 & $\mathbf{1 8}$ \\
Coxiella & 5 & 0 & 0 & $\mathbf{5}$ \\
Ehrlichia & 14 & 0 & 0 & $\mathbf{1 4}$ \\
Francisella & 13 & 0 & 0 & $\mathbf{1 3}$ \\
Rickettsia & 26 & 10 & 4 & $\mathbf{4 0}$ \\
Total & $\mathbf{8 1}$ & $\mathbf{1 3}$ & $\mathbf{8}$ & $\mathbf{1 0 2}$ \\
\hline Boldace & & &
\end{tabular}

Boldface used for emphasis pathway database. The final set comprised 102 genomes with a total of 120,046 protein sequences (Table 1, Additional file 1: Table S1). All complete genomes (plasmids were not used in this study) were downloaded via NCBI ftp service (ftp://ftp.ncbi.nlm.nih.gov/genomes) in August 2015. KEGG databases (http://www.genome.jp/ $\mathrm{kegg} /$ ) were accessed in October 2015 [19, 20].

A word on the notation used in the consecutive sections of this article. The pound sign and number following an organism name refer to the microorganism number in supplementary Table S1 (Additional file 1) and the number in the network in Fig. 1. For example, $R$. canadensis str. McKiel (\#39) indicates that the organism number is 39 both in the network of Fig. 1 and in Table S1 (Additional file 1).

The open-source GUI software package $p$ Clust and custom R scripts were used for phylogenomic network construction. $p$ Clust utilizes the Parasail package for fast sequence alignment [21, 22] and Grappolo for clustering [23]. Network visualization was done using the free software package visone [24]. Sequence alignment and clustering by means of $p$ Clust was performed on a desktop machine running Windows 8 with $8 \mathrm{~GB}$ of RAM and took less than 10 minutes for the 102 genomes. For more details and specific parameters for phylogenomic network construction and visualization as well as for information on downloading the software, consult Additional file 2.

\section{Results}

\section{Genome clustering}

Employing the $p$ Clust clustering procedure (Additional file 2), we found distinct cluster separation among different vector-borne organisms (Fig. 1). Overall, the network structure appears to be driven by divisions among different genera, but some important insights into relationships among the species are also provided. The tick-borne (white symbols) Borrelia (Fig. 1, squares top left), Francisella (Fig. 1, circles bottom left), and Ehrlichia (Fig. 1, squares far right) create clearly defined clusters with thicker meshes indicating closely related organisms. Within the Bartonella cluster (Fig. 1, diamonds left), a flea-borne (black symbols) group of bacteria is clearly defined (black diamonds). Tick-borne Anaplasma phagocytophilum forms a triangle (Fig. 1, trapezoids top right) independent from Ehrlichia while the A. marginale and A. centrale triangle (Fig. 1, trapezoids near right) is much closer to the Ehrlichia spp.

The order Rickettsiales was reorganized based on phylogenetic data from a few genes [25]. Our study is based on whole genome analysis. The genera Anaplasma and Ehrlichia have always been recognized as being more closely related to each other than to the other genera (Wolbachia and Neorickettsia) in the family. A. centrale is 


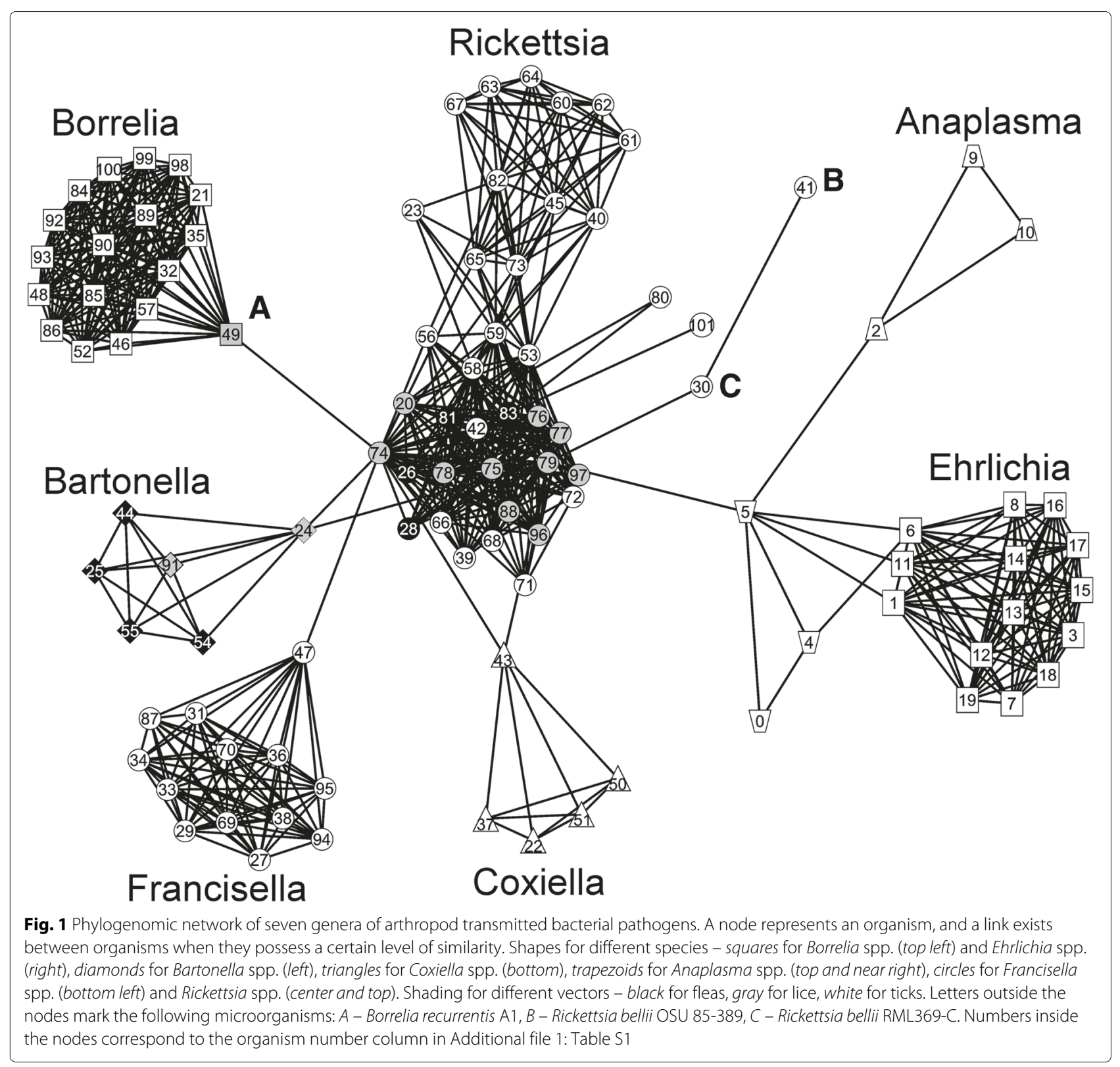

a subspecies of $A$. marginale, and therefore very closely related, so it makes sense that these would cluster very tightly. A. phagocytophilum has a larger genome than the other Anaplasma species and has an expansion of the $m s p 2$ superfamily. In addition, it contains more Ank domain effector molecules. These differences may be sufficient to set its genome/proteome apart given the very limited set of complete genomes examined. For example, there are no complete genomes available for A. platys or $A$. ovis, which, if available, might provide a slightly different picture of how these two genera cluster.

The Rickettsia cluster (Fig. 1, circles center and top) is not as compact as other clusters and several subgroups are distinguishable within it. Notably, it consists of the tick- borne $R$. rickettsii at the top, but the central core group contains in its center louse-borne (gray circles) and fleaborne (black circles) Rickettsia. It is interesting to note that Borrelia recurrentis (Fig. 1, A), a louse-transmitted species, which has a genome that appears to be a degraded subset of the tick-transmitted $B$. duttonii [26], serves as a transitional organism between the major body of the Rickettsia and Borrelia clusters. Also noteworthy is the independent positions of the R. bellii organisms (Fig. 1, B and $C$ ) which, together with $R$. canadensis (\#39 and \#66), are considered to form the ancestral group of Rickettsia [27]. While the organism clustering is genus-driven, it is intriguing that clusters emanating from the Rickettsia group are connected by their transitional organisms to a 
louse-borne species in the center of the Rickettsia cluster, i.e., the Bartonella, Borrelia, Francisella, Coxiella, and Ehrlichia (via Anaplasma spp.) clusters are connected to the Rickettsia cluster via louse-borne organisms (Fig. 1).

The origins and the ability of pathogens to be insectborne are also still largely unknown. Current evidence suggests that the genomes of louse-borne organisms are the result of degraded genomes from their arthropodborne relatives. For example, Borrelia duttonii, which is tick-borne, degraded to $B$. recurrentis which is louseborne with $20 \%$ genome reduction [26]; Bartonella henselae, a flea-borne pathogen, degraded to louse-borne $B$. quintana with $12.6 \%$ genome reduction [28]; and Rickettsia conorii (tick-borne) degraded to R. prowazekii (louse-borne) with $18 \%$ genome reduction [29]. Both louse- and flea-borne organisms are positioned in the center of the Rickettsia spp. cluster (Fig. 1), and many transitional genomes are connected to them. For example, Borrelia recurrentis (\#49), a louse-borne transitional organism for Borrelia spp., is connected to the louse-borne $R$. prowazekii str. GvV257 (\#74). Bartonella quintana str. Toulouse (\#24), a louse-borne transitional organism for Bartonella spp., and C. burnetii RSA 331 (\#43), a tick-borne transitional organism for Coxiella spp., are both connected to two louse-borne $R$. prowazekii strains - str. GvV257 (\#74) and str. RpGvF24 (\#79). At the same time the tick-borne $A$. centrale str. Israel (\#5), a transitional organism for Anaplasma spp., is connected only to the louse-borne $R$. prowazekii str. RpGvF24 (\#79).

\section{No genes are uniquely present in all tick-borne bacteria}

If a set of genes common to all tick-borne bacteria exists, then it implies there is a bio-molecular, tick-associated mechanism shared among all of them. Note that while we refer to homologous gene (HG) clusters, we actually clustered deduced amino acid sequences, or proteins; we use the terms synonymously. We examined the microbial genomes for homologous gene presence using 3D visualization (Fig. 2, Additional file 3: Figure S1). In Fig. 2, every ball represents a cluster of homologous genes, and its location in $(x, y, z)$ coordinates gives the fraction or percentage proportional to its appearance in the genome of an organism that is transmitted by one of the three vector types - ticks, lice, and fleas, respectively. For example, if a certain cluster is located at coordinates $(0.4,0.3,0.7)$, it means that the cluster appears in $40 \%$ of tick-borne organisms, $30 \%$ of louse-borne organisms, and $70 \%$ of flea-borne organisms in our set. Such a mapping allows quick identification of vector-specific clusters. For example, all homologous gene clusters unique only to tick-borne organisms would have coordinates $(x, 0,0)$, or, in other words, would lie on the tick-axis. Similarly, homologous gene clusters present exclusively in louse-

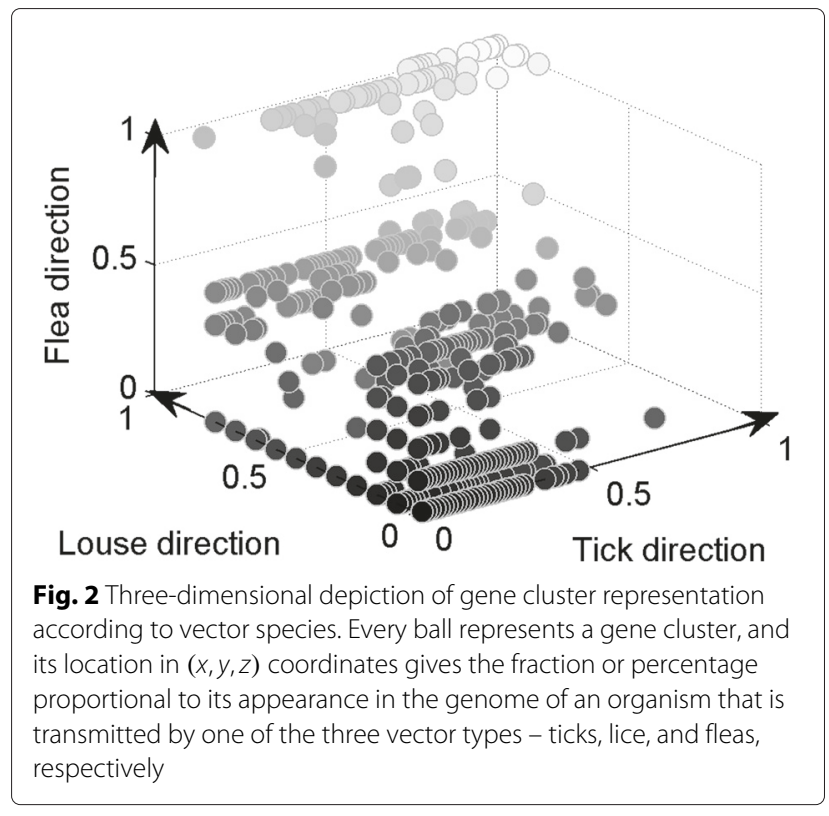

or flea-borne organisms would lie on the louse-axis and flea-axis, respectively. Homologous genes present in all organisms are located at $(1,1,1)$.

In Fig. 2 we observe that no gene cluster is at coordinates $(1,0,0)$, which indicates that there are no homologous genes present exclusively and entirely in all tick-borne organisms and, thus, no single set of genes is responsible for all tick-borne transmission. The same is true for both louse- and flea-borne organisms, although our sampling of these genomes is smaller. However, there are sets of homologous gene clusters that are unique to various subsets of tick-borne bacteria. To understand this, we constructed a multiplicity 3D plot in which the size of each ball indicates the number of the homologous gene clusters at that point (Additional file 3: Figure S1). For example, clusters that are present in few organisms are located closer to zero and they have high multiplicity as indicated by their sizes, i.e., balls are large. Because we are interested in tick transmissibility, we examine only the clusters located along the tick-axis. Figure 3 shows the multiplicity of each gene cluster along the tick-axis. For example, the last ball on the tick-axis (Additional file 3: Figure S1) corresponds to the last column in Fig. 3, from which we see that there are three different HG clusters present in 38 tick-borne organisms. Figure S2 (Additional file 3) shows exactly how the three clusters span the tick-borne organisms. In this case, the three HG clusters are present in closely related Anaplasma and Ehrlichia spp., but also in the more distant species of Francisella and Coxiella (Additional file 2: Figure S2). It is quite remarkable that organisms as different as Anaplasma and Francisella have common genes that are not present in any of the louseor flea-borne organisms. A similar situation is shown in 


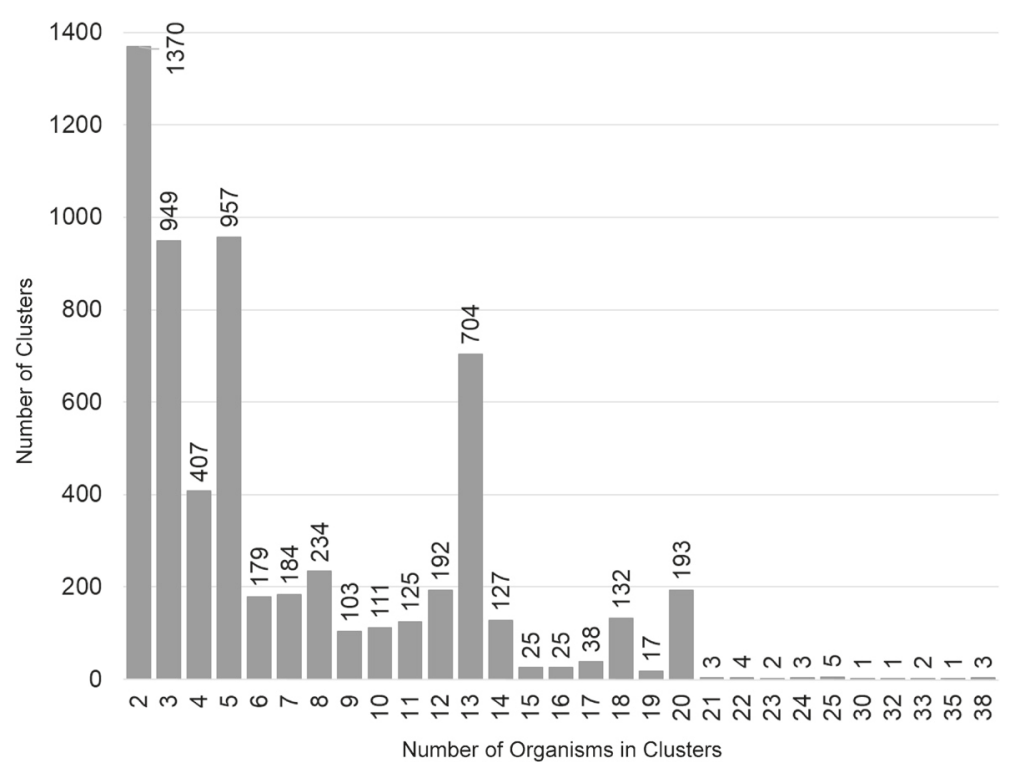

Fig. 3 Multiplicity plot of homologous gene clusters along the tick-axis. The bars represent the number of gene/protein clusters that are comprised of the number of tick-transmitted species represented on the $x$-axis, e.g., the rightmost bar indicates that there are 38 tick transmissible species that have three clusters in common (also see Additional file 3: Figure S2)

Figure S3 (Additional file 3) in which two HG clusters span three genera of tick-borne organisms - Anaplasma, Ehrlichia, and Francisella.

There are pathways present in sets of tick-borne bacteria that are absent from bacteria transmitted by lice or fleas We systematically examined various metabolic pathways associated with the tick-borne HG clusters spanning several genera. In particular, we examined all pathways associated with the last ten homologous gene clusters present in significant numbers in only tick-borne microorganisms. Table 2 details information for 25 unique HG clusters. While we did not find differences with respect to complete metabolic pathways, we did find several subpaths that are present in some genera while absent in others. In particular, we found that Anaplasma, Ehrlichia, Francisella, and Coxiella spp. all share parts of major metabolic pathways that are absent in any louse- or flea-borne bacteria.

Table 3 summarizes this information. The first example is a part of the nicotinate and nicotinamide metabolism pathway from L-asparate to nicotinate D-ribonucleotide (Table 3, Fig. 4a). Nicotinate and nicotinamide are the precursors for generation of coenzymes NAD+ and NADP+ $[30,31]$. These coenzymes are crucial for many metabolic pathways including glycolysis, TCA cycle, pentose phosphate cycle, fatty acid biosynthesis and metabolism pathways, and many others $[30,31]$. The path from L-asparate to nicotinate D-ribonucleotide is present in Anaplasma, Ehrlichia, Francisella, and Coxiella spp. - all tick-borne species (Fig. 4a). In Francisella and Coxiella this part of the pathway starts at L-asparate while in Anaplasma and Ehrlichia it starts from the freely available glycerone-P. The subpath is absent from Rickettsia and Borrelia spp., although some of these are tick-borne, as well as from Bartonella spp., which has only flea- and louse-borne organisms.

Another example is subpaths in the arginine and proline metabolism pathway (Fig. 4b-c). In this major pathway, bifunctional enzyme 1-pyrroline-5-carboxylate dehydrogenase (KEGG:EC 1.2.1.88) is responsible for a number of chemical reactions including 1) reversal from glutamate to L-glutamate 5-semialdehyde and back (Fig. 4b), 2) from 1-pyrroline-5-carboxylate to glutamate (Fig. 4b), and 3) from 1-pyrroline-3-hydroxy-5-carboxylate (Fig. 4c, path 3a) or 4-hydroxyglutamate semialdehyde (Fig. 4c, path 3b) to L-erythro-4-hydroxy-glutamate which further gets transformed into glyoxylate or pyruvate. These subpaths are present in Anaplasma, Ehrlichia, Francisella, and Coxiella spp. Neither Rickettsia nor Borrelia spp. can complete any of these while Bartonella spp. can only complete one-way conversion of subpath 1, i.e., from glutamate to L-glutamate 5-semialdehyde, but not back (Fig. 4b, gray arrows).

The absence of bifunctional 1-pyrroline-5-carboxylate dehydrogenase in Rickettsia, Borrelia, and Bartonella spp. causes disruption in a part of another major amino acid metabolic pathway, namely in the alanine, aspartate, and glutamate metabolism pathway (Fig. 4d). The pathway part - reversible conversion from L-1pyrroline-5-carboxylate to L-glutamate - is present in Anaplasma, Ehrlichia, Francisella, and Coxiella spp. while 
Table 2 Detailed information for the most widely present homologous gene clusters

\begin{tabular}{|c|c|c|c|c|}
\hline Cluster \# & $\begin{array}{l}\text { Representative } \\
\text { protein accession \# }\end{array}$ & $\begin{array}{l}\text { \# of organisms } \\
\text { in cluster }\end{array}$ & Product description & KEGG EC \# \\
\hline 30 & AAV86731 & 38 & $\begin{array}{l}\text { 2,3-bisphosphoglycerate-independent } \\
\text { phosphoglycerol mutase }\end{array}$ & 5.4.2.12 \\
\hline 30 & AAV87091 & 38 & $\begin{array}{l}\text { nicotinate-nucleotide } \\
\text { pyrophosphorylase }\end{array}$ & 2.4.2.19 \\
\hline 30 & AAV87100 & 38 & quinolinate synthetase A & 2.5.1.72 \\
\hline 29 & NP_212375 & 35 & glycerol kinase & 2.7.1.30 \\
\hline 28 & AAV86691 & 33 & $\begin{array}{l}\text { 1-deoxy-D-xylulose-5-phosphate } \\
\text { reductoisomerase }\end{array}$ & 1.1.1.267 \\
\hline 28 & AAV87137 & 33 & $\begin{array}{l}\text { 2-C-methyl-D-erythritol } \\
\text { 2,4-cyclodiphosphate synthase }\end{array}$ & 4.6.1.12 \\
\hline 27 & AHX03201 & 32 & $\begin{array}{l}\text { tRNA threonylcarbamoyladenosine biosynthesis } \\
\text { protein TsaE }\end{array}$ & no EC number \\
\hline 26 & NP_212377 & 30 & glycerol-3-phosphate dehydrogenase & 1.1.5.3 \\
\hline 25 & AAV87130 & 25 & hypothetical & no entry \\
\hline 25 & NP_359873 & 25 & hypothetical & no entry \\
\hline 25 & AAV86484 & 25 & pyridoxine 5-phosphate synthase & 2.6.99.2 \\
\hline 25 & AAV86592 & 25 & $\begin{array}{l}\text { bifunctional proline dehydrogenase, pyrroline-5- } \\
\text { carboxylate dehydrogenase }\end{array}$ & $1.5 .5 .2,1.2 .1 .88$ \\
\hline 25 & AAV86778 & 25 & thiamine biosynthesis oxidoreductase & 1.4.3.19 \\
\hline 24 & NP_359861 & 24 & divalent cation tolerance protein & no entry \\
\hline 24 & NP_360910 & 24 & hypothetical & no entry \\
\hline 24 & NP_360096 & 24 & hypothetical & no entry \\
\hline 23 & NP_360134 & 23 & hypothetical & no entry \\
\hline 23 & NP_360791 & 23 & hypothetical & no entry \\
\hline 22 & YP_001499275 & 22 & AbrB family transcriptional regulator & no entry \\
\hline 22 & NP_360166 & 22 & cell filamentation protein $\mathrm{Fic}^{\mathrm{a}}$ & no entry \\
\hline 22 & NP_360392 & 22 & hypothetical & no entry \\
\hline 22 & NP_360955 & 22 & plasmid stability protein & no entry \\
\hline 21 & NP_359844 & 21 & hypothetical & no entry \\
\hline 21 & NP_360418 & 21 & biotin-protein ligase & no entry \\
\hline 21 & NP_360956 & 21 & hypothetical & no entry \\
\hline
\end{tabular}

aThis gene was reported as one of three representative ORGs, RiOG_1005, i.e., present in all tick-borne Rickettsial genomes in the study by Gillespie et al. [27]

Table 3 Examples of pathways present in a subset of tick-borne genera

\begin{tabular}{|c|c|c|c|c|c|c|c|c|c|c|c|c|}
\hline \multirow[t]{2}{*}{ KEGG KO \#and pathway } & \multirow[t]{2}{*}{ Product } & \multicolumn{6}{|c|}{ Tick-borne } & \multicolumn{3}{|c|}{ Louse-borne } & \multicolumn{2}{|c|}{ Flea-borne } \\
\hline & & $A^{a}$ & $\mathrm{E}$ & $\mathrm{F}$ & C & Bo & $\mathrm{R}$ & $\mathrm{R}$ & Bo & $\mathrm{Ba}$ & $\mathrm{Ba}$ & $\mathrm{R}$ \\
\hline $\begin{array}{l}\text { 00760: Nicotinate and nicotinamide } \\
\text { metabolism }\end{array}$ & $\begin{array}{l}\text { nicotinate } \\
\text { D-ribonucleotide }\end{array}$ & + & + & + & + & - & - & - & - & - & - & - \\
\hline $\begin{array}{l}\text { 00250: Alanine, aspartate and glutamate } \\
\text { metabolism }\end{array}$ & various & + & + & + & + & - & - & - & - & - & - & - \\
\hline 00330: Arginine and proline metabolism & various & + & + & + & + & - & - & - & - & - & - & - \\
\hline
\end{tabular}

${ }^{\mathrm{a}}$ Genus abbreviations: $\mathrm{A}=$ Anaplasma; $\mathrm{E}=$ Ehrlichia; $\mathrm{F}=$ Francisella; $\mathrm{C}=$ Coxiella; $\mathrm{Bo}=$ Borrelia; $\mathrm{Ba}=$ Bartonella; $\mathrm{R}=$ Rickettsia 


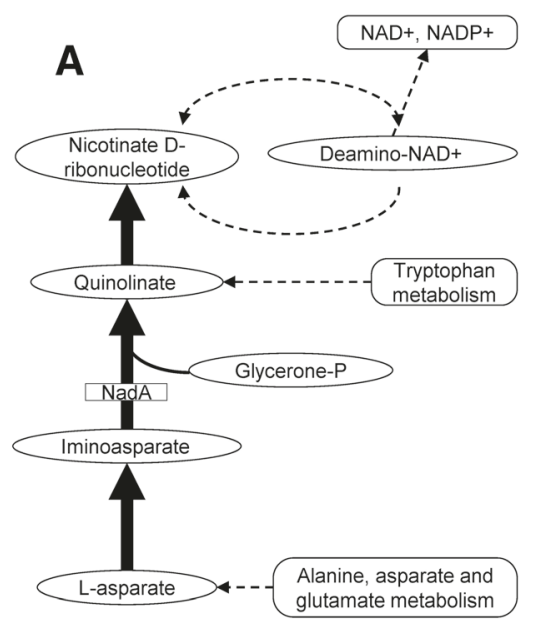

B
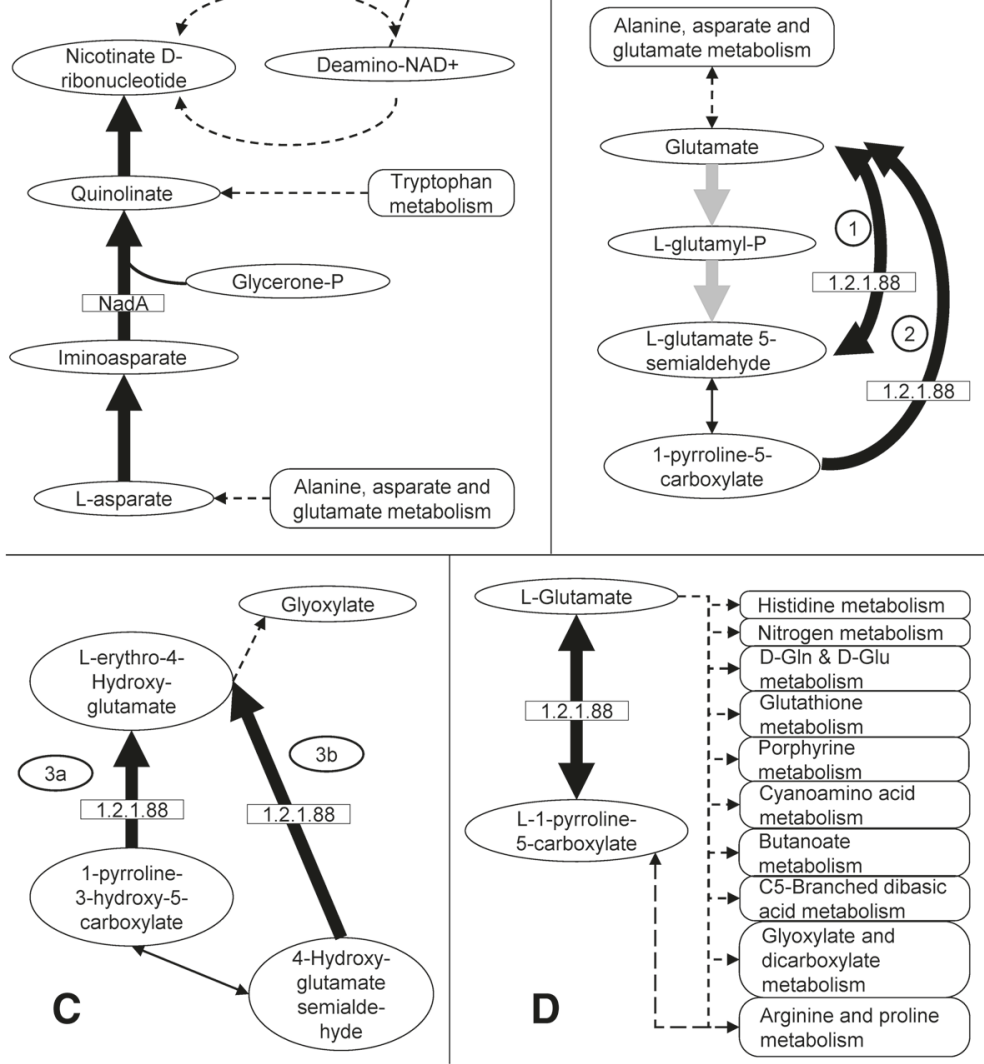

Fig. 4 Pathway analysis for different organisms. Thick black or gray arrows - enzyme-dependent subpaths; thin black arrows - enzyme-independent conversions; dashed arrows - other metabolic paths. Ovals - intermediate products; rectangles - enzymes of interest; rounded rectangles - major metabolic paths. a Part of nicotinate and nicotinamide metabolism pathway (KEGG:ko00760). This subpath is present in Anaplasma, Ehrlichia, Francisella, and Coxiella spp. In Francisella and Coxiella - from L-asparate to nicotinate D-ribonucleotide. In Anaplasma and Ehrlichia - from glycerone-P to nicotinate D-ribonucleotide. The path is absent in Bartonella, Rickettsia, and Borrelia spp. b, c Two subpaths of arginine and proline metabolism pathways (KEGG:ko00330). Both are present in Anaplasma, Ehrlichia, Francisella, and Coxiella spp. while absent in Rickettsia, Borrelia, and Bartonella spp., which can complete only the gray arrow path in B. d Part of alanine, aspartate, and glutamate metabolism pathway (KEGG:ko00250). Present in Anaplasma, Ehrlichia, Francisella, and Coxiella spp. while absent in Rickettsia, Borrelia, and Bartonella spp

Rickettsia, Bartonella, and Borrelia spp. do not have this subpath.

\section{Discussion}

We hypothesized that the ability of pathogens to be tick transmitted arose once in evolution. If this is correct, then we would expect to find a set of genes common to all tickborne bacteria and, presumably, these genes would confer tick transmissibility. To test this hypothesis, we selected a wide variety of vector-borne bacteria including species from multiple genera as well as non-tick-borne bacteria for contrast. To emphasize tick association, we looked at the set of homologous genes (HGs) that are present only in tick-borne pathogens and absent in flea- and louse-borne bacteria. We found that there are no tick-associated genes that are present in all tick-borne bacteria.
The absence of HGs unique to all tick-borne pathogens suggests their ability to be tick transmitted arose multiple times in evolution. An alternative possibility is that genes involved in the ability to be tick transmitted accumulated mutations to the extent that they are no longer recognized as homologs. Another possibility is that the ability of pathogens to be tick transmitted may not require a unique set of genes. However, if this were true, an organism with the basic set of essential genes would be tick transmissible, but this is not the case. Thus, given the absence of homologous genes shared by all tick-borne pathogens, we suggest that the ability of pathogens to be tick transmitted arose multiple times during their evolution, and different sets of organisms employ different sets of genes to confer this ability. We did find homologous genes that are common to different subsets of tick-borne organisms. These 
subsets consist of different combinations of Anaplasma, Ehrlichia, Francisella, and Coxiella spp. (Additional file 3: Figures S2, S3). Observe that no cross set of genes was found that included Rickettsia and other genera. This suggests parallel evolutionary development of tick association for Rickettsia and other species.

The existence of genes unique to tick-borne bacteria among the set of organisms analyzed does not necessarily suggest that the metabolic processes in which these genes are involved cannot be completed by other means, for example, either by employing a different enzyme or by completing a different pathway. However, the tickspecific homologous genes suggest where the tick-specific metabolic pathways might exist. Thus, we considered whether sufficient evidence could be detected for complete metabolic pathways by analyzing the pathways associated with the tick-specific HGs we identified. Because all organisms in our study are vector-borne, many of them have undergone reductive evolution and rely on the host cell environment to supply necessary substrates and chemical compounds [32-34]. Whether a pathway with few enzymes in it can be fully functional or not is unknown. Some experimental evidence exists showing that metabolic reactions can be successfully completed by harvesting necessary substrates from the host environment. For example, Frohlich et al. showed that while $R$. prowazekii is unable to synthesize DHAP as a substrate for the GpsA enzymatic reaction, it encodes and synthesizes a functional GpsA enzyme [35]. Therefore, in our pathway analysis rather than considering completion of a full metabolic pathway, we concentrated on whether or not a certain part, a subpath, of a metabolic pathway could be completed. In this way we found a few metabolic subpaths that are unique to tick-borne bacteria via the HGs associated with them.

Two of the subpaths are part of amino acid metabolic pathways and one is part of the nicotinate and nicotinamide metabolism pathway. Of particular interest is the latter pathway. Nicotinamidase, or PncA, is an enzyme involved in the production of NAD and was empirically shown to play an important role in the infectivity of Borrelia [36]. When a non-infectious, low-passage strain of B. burgdorferi was introduced with a copy of a gene coding for nicotinamidase on a shuttle vector, viable $B$. burgdorferi were recovered from all tissue samples [36]. Interestingly, the common subpath we found from L-asparate via quinolinate to nicotinate D-ribonucleotide (Fig. 4a) is present in Anaplasma, Ehrlichia, Francisella, and Coxiella spp. Thus, these organisms prefer an endogenous pathway for NAD synthesis while Rickettsia and Bartonella spp. must rely on other means such as, for example, in the case of Borrelia spp. which employ exogenous pathways for NAD synthesis [36].
We must note that our pathway analysis is not exhaustive. One reason for this is that functionality of many gene products is unknown and, thus, their participation in metabolic pathways cannot be defined. There may exist other sets of metabolic pathways common to tick- and other vector-borne pathogens, but their identification is hindered due to our limited knowledge. For example, 10 of 25 examined homologous genes do not have annotation in KEGG pathways because they are hypothetical (Table 2). Another reason is that while our knowledge of gene presence is complete - that is, there are only so many genes in a particular organism and, if a genome is sequenced, all of them are known - our current knowledge of metabolic pathways is far from complete. For example, an additional 4 of 25 examined homologous genes have no associated KEGG entry although their products are known (Table 2). This fact reflects the inherent complexity associated with biochemical processes in living organisms and our ongoing efforts to understand them.

It is interesting to note that just as there are no HGs common to all tick-borne pathogens, there are no HGs common to all louse- and flea-borne organisms as well. It is quite a remarkable fact given the relatively small set of organisms associated with these vectors examined in the present study. This is because the likelihood of organisms sharing common genes decreases as the size and diversity of the set grow. In the current set of organisms there are only 8 flea- and 13 louseassociated pathogens. Along the flea-axis (Fig. 2), the farthest point is located at $(0,0,0.5)$, which has multiplicity 20 meaning that $50 \%$, or equivalently 4 out of 8 , flea-borne organisms share 20 HGs. Close examination shows they are present only in flea-borne members of Bartonella (data not shown). A similar situation exists for louse-borne organisms. Along the louse-axis the farthest point is located at $(0,0.77,0)$, which has multiplicity 5 meaning that $77 \%$, or equivalently 10 out of 13 louse-borne organisms share 5 HGs and, again, all of them are present in only one genus, namely Rickettsia. Thus, in the case of louse- and flea-borne bacteria there are no genes that span multiple genera unlike the situation for tick-borne pathogens. In part this may be explained by the fact that we used only two genera with flea-borne organisms - Bartonella and Rickettsia - and three genera with louse-borne organisms - Borrelia, Bartonella, and Rickettsia. Future research may address this shortcoming.

\section{Conclusion}

We initiated our study with the hypothesis that there may exist a common molecular mechanism among all tick-associated pathogens. Such a common mechanism would greatly benefit our understanding of the 
pathogenicity of tick-borne microbial organisms. However, our analysis indicates that rather than having one molecular mechanism common to all tick-borne bacteria, there are several evolutionary paths that, perhaps, developed independently as different organisms spread into new hosts and acquired new niches. We also found a number of hypothetical gene products common to a significant number of organisms in our set. A tantalizing question remains open whether these genes hold the clues for molecular mechanisms specific to lifestyles within the arthropod host.

\section{Additional files}

Additional file 1: Organisms and their protein sequences. Table S1. Organisms and their protein sequence files in the current study. (PDF $385 \mathrm{~kb}$ )

Additional file 2: Network construction. Network construction methodology and baseline comparison study for network construction. (PDF $820 \mathrm{~kb}$ )

Additional file 3: Figure S1. Three-dimensional depiction of multiplicity of gene clusters according to vector species.

Figure S2. The span of three gene clusters across different tick-borne organisms. Figure S3. The span of two gene clusters across different tick-borne organisms. (PDF 606 kb)

\section{Abbreviations}

DHAP, dihydroxyacetone phosphate; GUI, graphical user interface; HG, homologous gene; KEGG, Kyoto Encyclopedia of Genes and Genomes; MB, megabyte; NAD, nicotinamide adenine dinucleotide; NADP, nicotinamide adenine dinucleotide phosphate; NCBI, national center for biotechnology information; RAM, random access memory; TBD, tick-borne disease.

\section{Acknowledgements}

We thank Prof. Ananth Kalyanaraman from Washington State University (WSU), School of Electrical Engineering and Computer Science (EECS), for his overall support with $p$ Clust and Dr. Jeff Daily (WSU, EECS) for helping with Parasail and Hao (Howard) Lu (WSU, EECS) for helping with Grappolo when the GUI pClust software tool was created by SL.

This work was funded by support from the National Science Foundation under the Advances in Biological Informatics program, Award 1262664.

\section{Availability of data and materials}

The datasets supporting the conclusions of this article are available for download via the NCBI ftp service ftp://ftp.ncbi.nlm.nih.gov/genomes and the protein sequence files are listed in Additional file 1.

Availability of the $p$ Clust software:

- Project name: pClust.

- Project home page: https://bitbucket.org/wsu_bcb/pclust/downloads.

- DOI: http://dx.doi.org/10.5281/zenodo.46483

- Operating system(s): Windows, Mac.

- Programming languages: Java, C, OpenMP.

- License: BSD 3-clause.

\section{Authors' contributions}

$S L, S L B, K A B$ conceived and designed the experiment. SL conducted the experiments and analyzed the data. SL, SLB, KAB drafted the manuscript. SLB and $K A B$ supervised the project. All authors have read and approved the final version of this manuscript.

\section{Competing interests}

The authors declare that they have no competing interests.

\section{Author details}

${ }^{1}$ School of Electrical Engineering and Computer Science, Washington State University, P.O. Box 642752, 99164-2752 Pullman, USA. ${ }^{2}$ Department of Veterinary Microbiology and Pathology, Washington State University, P.O. Box 647040, 99164-7040 Pullman, USA. ${ }^{3}$ Paul G. Allen School for Global Animal Health, Washington State University, PO Box 647090, $99164-7090$ Pullman, USA.

Received: 27 February 2016 Accepted: 14 May 2016

Published online: 02 July 2016

\section{References}

1. Using Climate to Predict Infectious Disease Outbreaks: a Review. World Health OrganizationGlobal Partnership to Roll Back Malaria. Accessed 1 Nov 2015. http://apps.who.int/iris/handle/10665/84175.

2. How Many People Get Lyme Disease? Centers for Disease Control and Prevention. Accessed 1 Nov 2015. http://www.cdc.gov/lyme/stats/ humancases.html.

3. Hepburn MJ, Simpson AJ. Tularemia: current diagnosis and treatment options. Expert Rev Anti-infective Therapy. 2008;6(2):231-40.

4. Duzlu O, Yildirim A, Inci A, Gumussoy KS, Ciloglu A, Onder Z. Molecular investigation of francisella-like endosymbiont in ticks and francisella tularensis in ixodid ticks and mosquitoes in turkey. Vector-Borne Zoonotic Dis. 2016;26(1):26-32.

5. Other Tick-borne Spotted Fever Rickettsial Infections. Centers for Disease Control and Prevention. Accessed 1 Nov 2015. http://www.cdc.gov/ otherspottedfever/.

6. Schwan TG, Piesman J. Vector interactions and molecular adaptations of lyme disease and relapsing fever spirochetes associated with transmission by ticks. Emerging Infect Dis. 2002;8(2):115-21.

7. Fraser CM, Casjens S, Huang WM, Sutton GG, Clayton R, Lathigra R, White O, Ketchum KA, Dodson R, Hickey EK, et al. Genomic sequence of a lyme disease spirochaete, borrelia burgdorferi. Nature. 1997;390(6660): $580-6$.

8. Hunter W, Kole C, Vol. 1. Genome Mapping and Genomics in Arthropods. Berlin: Springer; 2007

9. Chomel BB, Kasten RW, Floyd-Hawkins K, Chi B, Yamamoto K, Roberts-Wilson J, Gurfield AN, Abbott RC, Pedersen NC, Koehler JE. Experimental transmission of bartonella henselae by the cat flea. J Clin Microbiol. 1996;34(8):1952-6.

10. Higgins JA, Radulovic S, Jaworski DC, Azad AF. Acquisition of the cat scratch disease agent bartonella henselae by cat fleas (siphonaptera: Pulicidae). J Med Entomol. 1996;33(3):490-5.

11. Foil L, Andress E, Freeland RL, Roy AF, Rutledge R, Triche PC, O'Reilly KL. Experimental infection of domestic cats with bartonella henselae by inoculation of ctenocephalides felis (siphonaptera: Pulicidae) feces. J Med Entomol. 1998:35(5):625-8.

12. Finkelstein JL, Brown TP, O'reilly KL, Wedincamp J, Foil LD. Studies on the growth of bartonella henselae in the cat flea (siphonaptera: Pulicidae). J Med Entomol. 2002;39(6):915-9.

13. Foucault C, Brouqui $P$, Raoult $D$. Bartonella quintana characteristics and clinical management. Emerg Infect Dis. 2006;12(2):217-3.

14. Bacon RM, Kugeler KJ, Mead PS, et al. Surveillance for Lyme disease-United States, 1992-2006. Atlanta, GA: Department of Health \& Human Services, Centers for Disease Control and Prevention; 2008.

15. Lyme Disease Graphs. Centers for Disease Control and Prevention. Accessed 1 Nov 2015. http://www.cdc.gov/lyme/stats/graphs.html.

16. Stanek G, Wormser GP, Gray J, Strle F. Lyme borreliosis. The Lancet. 2012:379(9814):461-73

17. Adrion ER, Aucott J, Lemke KW, Weiner JP. Health care costs, utilization and patterns of care following lyme disease. PloS one. 2015;10(2):0116767.

18. Kivaria F. Estimated direct economic costs associated with tick-borne diseases on cattle in tanzania. Tropical Anim Health Product. 2006;38(4) 291-9.

19. Kanehisa M, Sato Y, Kawashima M, Furumichi M, Tanabe M. Kegg as a reference resource for gene and protein annotation. Nucleic Acids Res. 2016;44(D1):457-62.

20. Kanehisa M, Goto S. Kegg: kyoto encyclopedia of genes and genomes. Nucleic Acids Res. 2000;28(1):27-30

21. Daily J, Kalyanaraman A, Krishnamoorthy S, Vishnu A. A work stealing based approach for enabling scalable optimal sequence homology detection. J Parallel Distributed Comput. 2015;79:132-42. 
22. Daily JA. Scalable parallel methods for analyzing metagenomics data at extreme scale. PhD thesis: Washington State University, School of Electrical Engineering and Computer Science; 2015.

23. Lu H, Halappanavar M, Kalyanaraman A. Parallel heuristics for scalable community detection. Parallel Comput. 2015;47:19-37.

24. Nocaj A, Ortmann M, Brandes U. Untangling the hairballs of multi-centered, small-world online social media networks. J Graph Algorithms Appl. 2015;19(2):595-618. doi:10.7155/jgaa.00370.

25. Dumler JS, Barbet AF, Bekker C, Dasch GA, Palmer GH, Ray SC, Rikihisa $Y$, Rurangirwa FR. Reorganization of genera in the families rickettsiaceae and anaplasmataceae in the order rickettsiales: unification of some species of ehrlichia with anaplasma, cowdria with ehrlichia and ehrlichia with neorickettsia, descriptions of six new species combinations and designation of ehrlichia equi and'hge agent'as subjective synonyms of ehrlichia phagocytophila. Int J Syst Evol Microbiol. 2001;51(6):2145-65.

26. Lescot M, Audic S, Robert C, Nguyen T, Blanc G, Cutler SJ, Wincker P, Couloux A, Claverie JM, Raoult D, et al. The genome of borrelia recurrentis, the agent of deadly louse-borne relapsing fever, is a degraded subset of tick-borne borrelia duttonii. PLoS Genet. 2008;4(9):1000185.

27. Gillespie JJ, Williams K, Shukla M, Snyder EE, Nordberg EK, Ceraul SM, Dharmanolla C, Rainey D, Soneja J, Shallom JM, et al. Rickettsia phylogenomics: unwinding the intricacies of obligate intracellular life. PloS one. 2008;3(4):2018.

28. Alsmark CM, Frank AC, Karlberg EO, Legault BA, Ardell DH, Canbäck B, Eriksson AS, Näslund AK, Handley SA, Huvet M, et al. The louse-borne human pathogen bartonella quintana is a genomic derivative of the zoonotic agent bartonella henselae. Proc Nat Acad Sci USA. 2004;101(26): 9716-21.

29. Ogata H, Audic S, Renesto-Audiffren P, Fournier PE, Barbe $V$, Samson D, Roux V, Cossart P, Weissenbach J, Claverie JM, et al. Mechanisms of evolution in rickettsia conorii and r. prowazekii. Science. 2001;293(5537): 2093-8.

30. Kato M, Lin SJ. Regulation of nad+ metabolism, signaling and compartmentalization in the yeast saccharomyces cerevisiae. DNA Repair. 2014;23:49-58.

31. Dölle C, Rack JG, Ziegler M. Nad and adp-ribose metabolism in mitochondria. Febs J. 2013;280(15):3530-41.

32. Gottlieb Y, Lalzar I, Klasson L. Distinctive genome reduction rates revealed by genomic analyses of two coxiella-like endosymbionts in ticks. Genome Biol Evol. 2015;7(6):1779-96.

33. Andersson SG, Kurland CG. Reductive evolution of resident genomes. Trends Microbiol. 1998;6(7):263-8.

34. Darby AC, Cho NH, Fuxelius HH, Westberg J, Andersson SG. Intracellular pathogens go extreme: genome evolution in the rickettsiales. TRENDS Genet. 2007;23(10):511-20.

35. Frohlich KM, Roberts RA, Housley NA, Audia JP. Rickettsia prowazekii uses an sn-glycerol-3-phosphate dehydrogenase and a novel dihydroxyacetone phosphate transport system to supply triose phosphate for phospholipid biosynthesis. J Bacteriol. 2010;192(17): 4281-8.

36. Purser JE, Lawrenz MB, Caimano MJ, Howell JK, Radolf JD, Norris SJ. A plasmid-encoded nicotinamidase (pnca) is essential for infectivity of borrelia burgdorferi in a mammalian host. Mol Microbiol. 2003;48(3): 753-64.

\section{Submit your next manuscript to BioMed Central and we will help you at every step:}

- We accept pre-submission inquiries

- Our selector tool helps you to find the most relevant journal

- We provide round the clock customer support

- Convenient online submission

- Thorough peer review

- Inclusion in PubMed and all major indexing services

- Maximum visibility for your research

Submit your manuscript at www.biomedcentral.com/submit 